\title{
Normality of product spaces
}

by

\author{
Amer Bešlagić (Madison, Wi.) and Keiko Chiba (Ohya)
}

Abstract. Assume $X$ is hereditarily normal and hereditarily countably paracompact, $Y$ Lašnev and $G$ an open subspace of $X \times Y$. Then $G$ is normal iff $G$ is countably paracompact.

If furthermore $X$ is hereditarily paracompact (shrinking, collectionwise normal) then $G$ normal implies $G$ paracompact (shrinking, collectionwise normal).

Assume $X$ is a first countable, paracompact $P$-space and $Y$ a closed image of a normal $M$-space. Then $X \times Y$ is normal iff it is countably paracompact.

1. Introduction. By a space we mean a Hausdorff topological space and by a map we mean a continuous onto function.

Morita [20] proved the following (for the proof see [31] or [25]).

1.1. THEOREM [20]. Assume $X$ is normal, $M$ metric, and $X \times M$ countably paracompact. Then $X \times M$ is normal.

Then Rudin and Starbird [31] proved the converse; so we have

1.2. TheOREM $[20,31]$. Assume $X$ is normal and countably paracompact, and $M$ metric. Then $X \times M$ is normal iff $X \times M$ is countably paracompact.

Generalizing Theorem 1.1, Nagami [22] proved that $X \times M$ can be replaced by an open set $G \subset X \times M$, and Przymusiński [24] proved the converse; thus

1.3. THEOREM [22, 24]. Assume $X$ is hereditarily normal and hereditarily countably paracompact, $M$ metric, and $G$ an open subspace of $X \times M$. Then $G$ is normal iff $G$ is countably paracompact.

A Lašnev space is the closed image of a metric space. Hoshina [13] showed that the metric factor in Theorem 1.2 can be replaced by a Lašnev space. So the question whether the metric space in Theorem 1.3 can be replaced by a Lašnev space seems natural. Here we show that the answer to this question is yes (Corollary 3.6).

The concept of normal countably paracompact spaces can be generalized a little.

1.4. Definition. An open cover $\left\{V_{\alpha}: \alpha \in \varkappa\right\}$ is a shrinking of a cover $\left\{U_{\alpha}: \alpha \in \mathcal{X}\right\}$ iff for every $\alpha \in \chi, \bar{V}_{\alpha} \subset U_{\alpha}$.

A closed cover $\left\{V_{\alpha}: \alpha \in \varkappa\right\}$ is a closed shrinking of a cover $\left\{U_{x}: \alpha \in \varkappa\right\}$ iff for every $\alpha \in \varkappa, V_{\alpha} \subset U_{\alpha}$.

3 - Fundamenta Mathematicae 128. 2 
A space $X$ is $x$-shrinking iff every open cover of size $\leqslant x$ has a shrinking, and a space is shrinking iff it is $x$-shrinking for every $x$.

Note that a space is normal iff it is $n$-shrinking for all (one) finite $n>1$, and is normal and countably paracompact iff it is $\omega$-shrinking. Also, observe that if every open cover of size $\leqslant x$ has a closed shrinking the space is $x$-shrinking.

We know essentially only one example in ZFC of a normal, nonshrinking space, Rudin [26, 28]. Paracompact spaces, perfectly normal spaces are shrinking; normal $\theta$-refinable spaces (Yasui [33]) are shrinking. For more on this see $[29,30]$.

It is known that a normal product of a metric space and a shrinking space is shrinking [2], and Hoshina improved this to Lašnev spaces [14]. We show in Theorems 3.1 and 3.2 that the following holds.

1.5. THEOREM. Assume $X$ is hereditarily $x$-shrinking, $Y$ Lašnev, and $G$ an open subspace of $X \times Y$. Then $G$ is normal iff $G$ is $x$-shrinking.

In Section 4 we consider hereditarily collectionwise normal spaces and hereditarily paracompact spaces and show that a normal, open subspace of a product having one factor a Lašnev space and the other a hereditarily collectionwise normal space or hereditarily paracompact space is respectively collectionwise normal or paracompact (Theorems 4.1 and 4.3).

In Section 5 we consider $M$ and $P$ spaces. Recall that $\lambda^{\mu}$ denotes the set of all functions from $\mu$ to $\lambda$ and that $\lambda^{<\omega}=\bigcup\left\{\lambda^{n}: n \in \omega\right\}$. Also if $s \in \lambda^{\mu}$ then $\hat{s}\langle\alpha\rangle$ is an element of $\lambda^{\mu+1}$ defined by $\hat{s}\langle\alpha\rangle(\mu)=\alpha$ and $\hat{s}\langle\alpha\rangle \uparrow \mu=s$.

$M$ and $P$ spaces are introduced by Morita [19] as a generalization of metric spaces.

A space $X$ is an $M$-space iff there is a sequence $\left\langle\mathscr{U}_{m}: m \in \omega\right\rangle$ of locally finite open covers of $X$ satisfying the following condition: For every $x \in X$ and $\left\langle K_{m}: m \in \omega\right\rangle$ a decreasing sequence of nonempty closed subsets of $X$ with $K_{m} \subset \bigcap\left\{U \in \mathscr{U}_{m}: x \in U\right\}$ for $m \in \omega$, we have that $\bigcap_{m \in \omega} K_{m} \neq 0$.

A space $X$ is a $P$-space iff for every cardinal $\lambda$ and open family $\left\{H(s): s \in \lambda^{<\omega}\right\}$ such that $H(s) \subset H(\hat{s}\langle\alpha\rangle)$ for $\alpha \in \lambda$ there are closed sets $K(s) \subset H(s)$ for $s \in \lambda^{<\omega}$ such that $U K(f \uparrow n)=X$ whenever $U H(f \uparrow n)=X$ for $f \in \lambda^{\omega}$.

$n \in \omega$
rita showed [19] that $X$ is a normal $P$-space iff $X \times M$ is normal for every metric space $M$.

We show that the product of a first countable, paracompact $P$-space with the closed image of a normal $M$-space is normal iff it is countably paracompact (Corollary 5.9).

Concerning the results in Section 5 it should be noted that the product of a first countable, paracompact $P$-space and a normal $M$-space is always normal [6, Theorem 5].

Some conditions on factors are necessary for each of our theorems. It is well known that there is a non-normal countably paracompact product of two normal spaces, for example $\omega_{1} \times\left(\omega_{1}+1\right)$. Also, $\diamond$ implies that there is a normal countably paracompact (shrinking) space $X$ such that $X^{2}$ is normal but not countably paracompact [3].

For all the facts and notions that are used without mention we refer the reader to [11]. Many more results about normality in products can be found in [25], and for all kinds of generalized metric spaces the reader can see [12].

2. Preliminaries. Here we fix our notation and state some facts that are used in the next sections.

Two notations for the closure operator are used, $\bar{A}$ and $\mathrm{cl} A$. The bar notation is used to denote the closure with respect to the largest space, so if $A \subset B \subset X$ then $\bar{A}=\mathrm{cl}_{X} A$. To avoid confusion sometimes we use $\mathrm{cl}_{X} A$ instead of $\bar{A}$.

If $\mathscr{F}$ is a family of sets and $X$ an arbitrary set then

$$
\operatorname{St}(X, \mathscr{F})=\bigcup\{F \in \mathscr{F}: F \cap X \neq 0\} \text {. }
$$

If $f$ is a function from $X$ to $Y$ and $A$ a subset of $X$ then $f^{\#}(A)=Y \backslash f(X \backslash A)$.

2.1. Lemma [15]. Let $f$ be a closed map from a normal $M$-space $X$ onto a space $Y$. Then there are disjoint sets $Y_{i}$, for $i \in \omega$, such that $Y=\bigcup_{i \in \omega} Y_{i}, Y_{0}=\left\{y \in Y: f^{-1}(y)\right.$ is countably compact $\}$, and $Y_{i}$ is closed and discrete for $i>0$.

In particular, if $X$ is metric (and hence an $M$-space) this is due to Lašnev [17], and in that case $Y_{0}=\left\{y \in Y: f^{-1}(y)\right.$ is compact $\}$ and $Y_{0}$ is metrizable.

A cover $\left\{V_{\alpha}^{n}: \alpha \in x \wedge n \in \omega\right\}$ is a (closed) $\sigma$-shrinking of a cover $\left\{U_{\alpha}: \alpha \in x\right\}$ iff each $V_{\alpha}^{n}$ is open (closed) and $\overline{V_{\alpha}^{n}} \subset U_{\alpha}\left(V_{\alpha}^{n} \subset U_{\alpha}\right)$.

2.2. LEMMA [2]. A space is $\varkappa$-shrinking iff every open cover of size $\leqslant \varkappa$ has a $\sigma$-shrinking.

2.3. Lemma [13]. Assume $X$ is countably paracompact, $A$ a closed subset of $X$, and $\left\{U_{n}: n \in \omega\right\}$ an open family such that $A \subset \cup U_{n}$ and $B \cap \bar{U}_{n}=0$ for $n \in \omega$. Then there are disjoint open $U, V$ with $A \subset U$ and $B \subset V$.

2.4. LEMMA. If $\left\{U_{\alpha}^{n}: \alpha \in \varkappa \wedge n \in \omega\right\}$ is an open family such that for $\alpha \in \varkappa$, $H_{\alpha} \subset \bigcup_{n \in \omega} U_{\alpha}^{n}$ and $H_{\alpha} \cap \overline{\bigcup_{\beta \neq \alpha} U_{\beta}^{n}}=0$ for $n \in \omega$, then there is an open family $\left\{U_{\alpha}: \alpha \in \mathcal{X}\right\}$ of pairwise disjoint sets with $H_{\alpha} \subset U_{\alpha}$ for $\alpha \in \varkappa$.

2.5. LEMMA [23]. Every normal $M$-space is collectionwise normal.

A space $X$ is a $\Sigma$-space iff there is a sequence $\left\langle\mathscr{F}_{m}: m \in \omega\right\rangle$ of locally finite closed covers of $X$ satisfying the following condition: For every $x \in X$ and $\left\langle K_{m}: m \in \omega\right\rangle$ a decreasing sequence of nonempty closed subsets of $X$ with $K_{m} \subset \bigcap\left\{F \in \mathscr{F}_{m}: x \in F\right\}$ for $m \in \omega$, we have $\bigcap_{m \in \omega} K_{m} \neq 0$.

2.6. LeMMA [21]. Every $M$-space is a $\Sigma$-space.

2.7. Lemma [21]. Assume $X$ is a $\Sigma$-space. Then there is a family $\left\{\mathscr{F}_{m}: m \in \omega\right\}$ of locally finite closed covers of $X$ and a cardinal $\lambda$ such that

(i) $\mathscr{F}_{m}=\left\{F(s): s \in \lambda^{m}\right\}$.

(ii) Every $F(s)=\bigcup\left\{F\left(s^{\hat{\alpha}}\langle\alpha\rangle\right): \alpha \in \lambda\right\}$. 
For $x \in X$ and $m \in \omega$ let $C\left(x, \mathscr{F}_{m}\right)=\bigcap\left\{F \in \mathscr{F}_{m}: x \in F\right\}$ and $C(x)=\bigcap_{m \in m} C\left(x, \mathscr{F}_{m}\right)$. And call a farnily $\mathscr{F}$ a net for $C(x)$ iff for every open $U \supset C(x)$ ther' is an $F \in \mathscr{T}$ with $C(x) \subset F \subset U$. Then

(iii) For every $x \in X$ there is an $f \in \lambda^{(\omega)}$ such that $\{F(f \uparrow n): n \in(0)$ is a net for $C(x)$.

(iv) $C(x)$ is countably compact.

2.8. Lemma [23]. Assume $X$ is a first countable space and $f: Z \rightarrow Y$ is a closed map with $f^{-1}(y)$ countably compact for $y \in Y$. Then $\left(\operatorname{id}_{x} \times f\right): X \times Z \rightarrow X \times Y$ is closed.

2.9. Lemma [7]. Assume $X$ is a first countable paracompact P-space and $Y$ a collectionwise normal $\Sigma$-space. Then $X \times Y$ is collectionwise normal.

A space is subparacompact iff every open cover has a $\sigma$-discrete closed refinement.

In what follows $x$ is always an infinite cardinal

3. Products with a Lašnev factor. We consider open subspaces of the products having one factor a Lašnev space. Our theorems are more general and the proofs shorter than the ones in [13] and [24].

3.1. THEOREM. Assume $X$ is hereditarily $x$-shrinking, $Y$ Lašnev, and $G$ an open subset of $X \times Y$. If $G$ is normal then $G$ is $x$-shrinking.

Proof. Let $\mathscr{U}=\left\{U_{\alpha}: \alpha \in \varkappa\right\}$ be an open cover of G. We find a $\sigma$-shrinking of $\mathscr{U}$, thus showing that $G$ is $\varkappa$-shrinking.

Let $Y=\bigcup_{i \in \omega} Y_{i}$ where $Y_{0}$ is metrizable and each $Y_{i}$, for $i>0$, is closed discrete (Lemma 2.1)

Since $X$ is hereditarily $x$-shrinking, $G$ normal, and $Y_{i}, i>0$, closed discrete, we can find, for every $\alpha \in \chi$, an open (in $G) U_{\alpha}^{i}$ with $\left(X \times Y_{i}\right) \cap G \subset U\left\{U_{\alpha}^{i}: \alpha \in \chi_{\text {; }}\right.$ and $\operatorname{cl}_{G} U_{\alpha}^{i} \subset U_{\alpha}$. Let $U=\bigcup\left\{U_{\alpha}^{i}: \alpha \in \varkappa \wedge i>0\right\}$; so $U$ is an open subset of $G$ covering $\left(X \times \bigcup_{i>0} Y_{i}\right) \cap G$.

It remains to cover $G \backslash U$. Let $Y_{0}^{\prime}=\left\{y \in Y_{0}: y\right.$ is non-isolated in $\left.Y_{0}\right\}$; sinee $Y_{0} \backslash Y_{0}^{\prime}$ is a countable union of closed discrete subsets of the metrizable space $Y_{0}$, it is easy to find a family $\left\{A_{\alpha}^{i}: \alpha \in \varkappa \wedge i \in \omega\right\}$ of closed subsets of $\left(X \times Y_{0}\right) \cap G$ such that for every $\alpha \in \chi$ and $i \in \omega, A_{\alpha}^{i} \subset U_{\alpha}$ and $U\left\{A_{\alpha}^{i}: \alpha \in \chi \wedge i \in \omega\right\}=\left(X \times\left(Y_{0} \backslash Y_{0}^{\prime}\right)\right) \cap G$. Then $\left\{A_{\alpha}^{i} \backslash U: i \in \omega \wedge \alpha \in x\right\}$ is a family of closed subsets of $G$ covering $(G \backslash U) \cap$ $\cap\left(X \times\left(Y_{0} \backslash Y_{0}^{\prime}\right)\right)$. By the normality of $G$ we can easily extend these sets to open sets whose closures lie inside the appropriate $U_{\alpha}$ 's.

Now we have to deal with $Y_{0}^{\prime}$, and we again use the fact that $Y_{0}$ is metrizable. Let $\mathscr{B}=\bigcup\left\{\mathscr{B}_{n}: n \in \omega\right\}$ be a basis for $Y_{0}$ such that for every $n, \mathscr{B}_{n}$ is a locally finite open cover of $Y_{0}$ with sets of diameter $<\frac{1}{2^{n}}$. For $n \in \omega$, let $: \mathscr{B}_{n}^{\prime}=\left\{B \in \mathscr{B}_{n}: B\right.$ is nondiscrete in $\left.Y_{0}\right\}$, and let $\mathscr{B}^{\prime}=\bigcup\left\{\mathscr{B}_{n}^{\prime}: n \in(\omega)\right.$. By induction, for every $B \in \mathscr{B}^{\prime}$ pick two points $p_{B}, q_{B} \in B$ so that no point of $Y_{0}$ is picked twice.
For $B \in \mathscr{B}^{\prime}$ and $\alpha \in \varkappa$, let $O_{B, \alpha}$ be the maximal open set in $X$ with $O_{B, \alpha} \times B \subset U_{\alpha}$, and define $O_{B}=\bigcup\left\{O_{B, \alpha}: \alpha \in \mathcal{X}\right\}$ and $P_{B}=X \backslash O_{B}$.

For $B \in \mathscr{B}_{n}^{\prime}$ and $m \geqslant n$ define

$$
K_{n}^{B}=\bigcup\left\{\left[\left(P_{B} \backslash \bigcup\left\{O_{C}: C \subset B \wedge C \in \bigcup\left\{\mathscr{B}_{k}^{\prime}: n \leqslant k<m\right\}\right\}\right) \cap \bar{O}_{D}\right] \times\left\{p_{D}\right\}:\right.
$$

and

$$
\left.D \subset B \wedge D \subset \mathscr{B}_{m}^{\prime}\right\}
$$

$$
H_{m}^{B}=\bigcup\left\{\left[\left(P_{B} \backslash \bigcup\left\{O_{C}: C \subset B \wedge C \in \bigcup\left\{\mathscr{B}_{k}^{\prime}: n \leqslant k<m\right\}\right\}\right) \cap \bar{O}_{D}\right] \times\left\{q_{D}\right\}:\right.
$$

$$
\left.D \subset B \wedge D \in \mathscr{B}_{m}^{\prime}\right\} \text {. }
$$

Observe that both $K_{m}^{B}$ and $H_{m}^{B}$ are closed in $X \times Y_{0}$ since the families $\left\{p_{D}\right.$ : $\left.D \in: \mathscr{B}_{m}^{\prime}\right\}$ and $\left\{q_{D}: D \in \mathscr{B}_{m}^{\prime}\right\}$ are locally finite.

Let $K^{B}=\bigcup_{m \geqslant n} K_{m}^{B}$ and $H^{B}=\bigcup_{m \geqslant n} H_{m}^{B}$. Now we check that $K^{D} \cap G$ and $H^{B} \cap G$ are closed subsets of $\left(X \times Y_{0}\right) \cap G$. Fix $\langle x, y\rangle \in\left(X \times Y_{0}\right) \cap G \backslash K^{B}$; enough to find

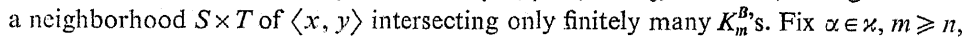
and a neighborhood $S \times T$ of $\langle x, y\rangle$ such that for every $l \geqslant m$ and $C \in \mathscr{B}_{l}$, if $C \cap T \neq 0$ then $S \times C \subset U_{\alpha}$ (this can be done since the diameters of the members of $\mathscr{H}_{l}$ are less than $\left.\frac{1}{2^{l}}\right)$. If $(S \times T) \cap K_{l}^{B} \neq 0$ for some $l \geqslant m$, there is a $C \in \mathbb{B}_{l}^{\prime}$ with $C \subset B$ and $p_{C} \in T$, so $S \times C \subset U_{\alpha}$ implying $S \subset O_{C}$; hence $(S \times T) \cap\left(\bigcup_{t>l} K_{t}^{B}\right)=0$.

Let $K=\left(\bigcup\left\{K^{B}: B \in \mathscr{B}^{\prime}\right\}\right) \cap G$ and $H=\left(\bigcup\left\{H^{B}: B \in \mathscr{B}\right\}\right) \cap G$. Then an argument similar to the one in the preceeding paragraph shows that $H$ and $K$ are closed subsets of $\left(X \times Y_{0}\right) \cap G$, so, since $H$ and $K$ are disjoint, $\operatorname{cl}_{G} H \cap \mathrm{cl}_{G} K \subset U$. There is a $T$, open in $G$, such that $\mathrm{cl}_{G} H \cap \mathrm{cl}_{G} K \subset T \subset \mathrm{cl}_{G} T \subset U$. Since $K \backslash T$ and $H \backslash T$ are closed disjoint subsets of $G$, there are $V, W$, open in $G$, with $\mathrm{cl}_{G} V \cap \mathrm{cl}_{G} W=0$, and $K \backslash T \subset V, H \backslash T \subset W$.

Let $\pi: X \times Y \rightarrow X$ be the projection map. For $n \in \omega$ and $B \in \mathscr{B}_{n}^{\prime}$, define

$$
F_{B}=(X \backslash \pi((X \times B) \cap T) \backslash(\pi((X \times B) \cap V) \cap \pi((X \times B) \cap W)),
$$

and note that $F_{B}$ is a closed subset of $X$. We now show that

$$
O_{B} \times\left(B \cap Y_{0}^{\prime}\right) \supset\left(F_{B} \times\left(B \cap Y_{0}^{\prime}\right)\right) \cap(G \backslash T) .
$$

To see this let $\langle x, y\rangle \in\left(F_{B} \times\left(B \cap Y_{0}^{\prime}\right)\right) \cap G$; then $\{x\} \times B \cap T=0$. Assume $x \notin O_{B}$ and show $x \notin F_{B}$ obtaining a contradiction. Let $m \geqslant n$ be the least such that there is a $C \in \mathscr{B}_{m}^{\prime}$ with $C \subset B$ and a neighborhood $S$ of $x$ with $S \times C \subset U_{\alpha}$ for some $\alpha$. Such $C$ exists since $y \in Y_{0}^{\prime}$; then $K \cap(\{x\} \times C) \neq 0$ and $H \cap(\{x\} \times C) \neq 0$, and $K \cap(\{x\} \times C) \cap T=0, \quad H \cap(\{x\} \times C) \cap T=0$, hence $x \in \pi((X \times B) \cap V) \cap$ $\pi((X \times B) \cap W)$.

Recall that $X$ is hereditarily $x$-shrinking. For $B \in \mathscr{B}^{\prime}$ let $\left\{Q_{B, \alpha}: \alpha \in \varkappa\right\}$ be a family 
of closed subsets of $O_{B} \cap F_{B}$ such that for each $\alpha, Q_{B, \alpha} \subset O_{B, \alpha}$, and $O_{B} \cap F_{B}$ $=\bigcup\left\{Q_{B, \alpha}: \alpha \in \mathcal{X}_{j}\right.$. Also, for each $n$, let $\left\{C_{B}: B \in \mathscr{B}_{n}\right\}$ be a closed cover of $Y_{0}$ with $C_{B} \subset B$ for $B \in \mathscr{B}_{n}$. For $\alpha \in \mathcal{X}$ and $n \in \omega$ define

$$
D_{x, n}=\bigcup\left\{Q_{B, \alpha} \times C_{B}: B \in \mathscr{P}_{n}^{\prime}\right\} \cap(G \backslash U) \cap\left(X \times Y_{0}^{\prime}\right) .
$$

To finish the proof it is enough to verify (by Lemma 2.2)

(i) for each $\alpha, n, D_{\alpha, \eta} \subset U_{\alpha,}$,

(ii) each $D_{\alpha, n}$ is closed in $G$,

(iii) $\bigcup\left\{D_{\alpha, n}: \alpha \in \chi \wedge n \in \omega\right\}=(G \backslash U) \cap\left(X \times Y_{0}^{\prime}\right)$.

Since (i) is trivial, we first show (ii). Fix $\alpha, n$ and $\langle x, y\rangle \in \mathrm{cl}_{G} D_{\alpha, n}$. Then $\langle x, y\rangle \in G \backslash U$ and $y \in Y_{0}^{\prime}$. Since $\mathscr{B}_{n}^{\prime}$ is locally finite in $Y_{0}$, there is a $B \in \mathscr{B}_{n}^{\prime}$ with $\langle x, y\rangle \in \operatorname{cl}_{G}\left(Q_{B, \alpha} \times C_{B}\right)$. Since $F_{B}$ is closed in $X$ and $Q_{B, \alpha} \subset F_{B}, x \in F_{B}$. But then $\langle x, y\rangle \in\left(F_{B} \times\left(B \cap Y_{0}^{\prime}\right)\right) \cap(G \backslash T)$ (since $\left.T \subset U\right)$, so $x \in O_{B}$ by (*); hence $x \in Q_{B, \alpha}, Q_{B, \alpha}$ being closed in $F_{B} \cap O_{B}$, so $\langle x, y\rangle \in Q_{B, \alpha} \times C_{B}$.

Ad (iii), let $\langle x, y\rangle \in(G \backslash U) \cap\left(X \times Y_{0}^{\prime}\right)$ and assume that $\langle x, y\rangle \phi \mathrm{cl}_{G} V$. Fix an $n$ and $S$ a neighborhood of $x$ in $X$ such that there is an $\alpha$ with

$$
S \times\left(\bigcup\left\{B \in \mathscr{B}_{n}: y \in B\right\}\right) \subset U_{\alpha} \backslash\left(\mathrm{cl}_{G} T \cup \mathrm{cl}_{G} V\right) .
$$

So for each $B \in \mathscr{B}_{n}^{\prime}$ with $y \in B$ we have that $x \in F_{B} \cap O_{B}$; hence there is a $B \in \mathscr{B}_{n}^{\prime}$ with $y \in C_{B}$ and a $\beta \in \chi$ with $x \in Q_{B, \beta}$. So $\langle x, y\rangle \in D_{\beta, n}$.

3.2. THEOREM. Assume $X$ is hereditarily normal, $Y$ Lašnev, and $G$ an open subset of $X \times Y$. If $G$ is countably paracompact then $G$ is normal.

Proof. Let $M$ be metric and $f$ a closed map from $M$ onto $Y$. By Lemma 2.1, $Y=\bigcup_{i \geqslant 0} Y_{i}$ such that $Y_{0}=\left\{y \in Y: f^{-1}(y)\right.$ is compact $\}$ and each $Y_{i}$ is closed discrete for $i>0$. Now we need some lemmas. 3.3. LemMa. If $A, B$ are closed disjoint subsets of $G$ such that $B \subset X \times\left(\bigcup_{i>0} Y_{i}\right)$,
then $A$ and $B$ are separated by open sets.

Proof. Use Lemma 2.3 twice. For $i>0$ and $y \in Y_{i}$ fix open $U_{y}^{n}, n \in \omega$, containing $y$ and $\left\{U_{y}^{n}: n \in \omega\right\}$ is decreasing, so that $\left\{U_{y}^{n}: y \in Y_{l}\right\}$ is discrete and $\left\{y_{\}}\right.$ $=\bigcap\left\{\mathrm{cl}_{Y} U_{y}^{n}: n \in \omega\right\}$. Also let $V_{y}$ be an open subset of $X$ with $B \cap(X \times\{y\})=V_{y} \times$ $\times\{y\}$ and $\left[\left(\mathrm{cl}_{x} V_{y}\right) \times\{y\}\right] \cap A=0$. If $W_{n}=\bigcup\left\{V_{y} \times U_{y}^{n}: y \in Y_{i}\right\} \cap G$ we have that $\left(\bigcap_{n \in \omega} \mathrm{cl}_{G} W_{n}\right) \cap A=0$, so by Lemma 2.3 there is an open $V_{i} \supset B \cap\left(X \times Y_{i}\right)$ with

$A \cap \mathrm{cl}_{G} V_{i}=0$. Lemma 2.3 applied again shows $A, B$ are separated by open sets.

3.4. LEMmA. If $A, B$ are closed disjoint subsets of $G$ such that $A \cup B \subset X \times Y_{0}$. then $A, B$ can be separated by open sets.

Proof. For $n \in \omega$, let $\mathscr{C}_{n}$ be a family of open subsets of $Y$ covering $Y_{0}$ such that $\mathscr{C}_{n}$ is locally finite in $U \mathscr{C}_{n}$ and each $\mathscr{C}_{n+1}$ refines $\mathscr{C}_{n}$. Also assume that for every $y \in Y_{0}$ and $V$ an open set in $Y$ containing $y$ there are $n \in \omega$ and $W$, open in $Y$, with $y \in W$ and $\operatorname{St}\left(W, \mathscr{C}_{n}\right) \subset V$. Let $\mathscr{C}=\bigcup \mathscr{C}_{n}$
To see that we can get the $\mathscr{C}_{n}$ 's, for $y \in Y_{0}$ let $V_{y}=\left\{m \in M: d\left(m, f^{-1}(y)\right)<\frac{1}{2^{n}}\right\}$. Then since $Y$ is hereditarily paracompact let $\mathscr{C}_{n}$ be a suitable refinement of $\left\{Y \backslash f\left(X \backslash V_{y}\right): y \in Y_{0}\right\}$ covering $\bigcup\left\{Y \backslash f\left(X \backslash V_{y}\right): y \in Y_{0}\right\}$.

For $C \in \mathscr{C}$ let $O_{C}$ be the maximal open set in $X$ with $O_{C} \times \bar{C} \subset G$. Let $\pi: X \times Y \rightarrow X$ be the projection map and define

$$
F_{C}=\left[\left(\left(X \backslash O_{C}\right) \times \bar{C}\right) \cup(\overline{(\pi(A \cap(X \times \bar{C}))} \cap \overline{\pi(B \cap(X \times \bar{C}))}) \times \bar{C}\right] \cap G .
$$

For $n \in \omega$, let $F_{n}=\bigcup\left\{F_{C}: C \in \mathscr{C}_{n}\right\}$.

We now show that $\left(\bigcap_{n \in \omega} \mathrm{cl}_{G} F_{n}\right) \cap\left(X \times Y_{0}\right)=0$. To see this let $\langle x, y\rangle$ $\epsilon\left(X \times Y_{0}\right) \cap G$ and let $V \times W \subset G$ be an open neighborhood of $\langle x, y\rangle$ not intersecting one of $A, B$, say $A$. Fix $n \in \omega$ and $W_{0}$, an open neighborhood of $y$, such that $\bigcup\left\{\bar{C}: C \in \mathscr{C}_{n} \wedge \bar{C} \cap W_{0} \neq 0\right\} \subset W$ (here we use the fact that $Y$ is regular and that there are $n \in \omega$ and $W_{0}$ containing $y$ with $\left.\operatorname{St}\left(W_{0}, \mathscr{C}_{n}\right) \subset W\right)$. This shows $\left(V \times W_{0}\right) \cap F_{n}=0$ so $\langle x, y\rangle \notin \mathrm{cl}_{G} F_{n}$.

Using Lemma 3.3 fix an open set $V$ in $G$ such that $\bigcap_{n \in \infty} \mathrm{cl}_{G} F_{n} \subset V$ and $\left(\operatorname{cl}_{G} V\right) \cap(A \cup B)=0$. Since each $\mathscr{C}_{n+1}$ refines $\mathscr{C}_{n}$, we get that $F_{n+1} \subset \omega$ So, by the countable paracompactness of $G$, there is a sequence $\left\langle U_{n}: n \in \omega\right\rangle$ of open subsets of $G$ such that $\bigcap_{n \in \omega} \mathrm{cl}_{G} U_{n}=0$ and $\operatorname{cl}_{G} F_{n} \backslash V \subset U_{n}$ for $n \in \omega$.

Fix $n \in \omega$ and $m \geqslant n$. For $C \in \mathscr{C}_{m}$ the set $E_{C}=X \backslash \pi\left(\left(V \cup U_{n}\right) \cap(X \times \bar{C})\right)$ is closed in $X$, and we show that

$$
\left.\left[\overline{\left(\pi \left(A \cap\left(E_{C} \times \bar{C}\right)\right.\right.}\right) \cap \overline{\pi(B \cap(X \times \bar{C}))) \times \bar{C}}\right] \cap G=0 .
$$

To see this observe that if $\langle x, y\rangle$ is the above intersection then

$$
\langle x, y\rangle \in F_{C} \subset F_{m} \subset U_{n} \cup V \text { so } x \notin E_{C} .
$$

Let $Z=X \backslash \overline{\left(A\left(A \cap\left(E_{C} \times \bar{C}\right)\right)\right.} \cap \overline{\pi(B \cap(X \times \bar{C})))}$. Since $Z$ is normal, let $D_{C, n}$ be an open set in $Z$ (hence in $X$ ) such that $Z \cap \overline{\pi\left(A \cap\left(E_{C} \times \bar{C}\right)\right)} \subset D_{C, n}$ and $\left(\mathrm{cl}_{Z} D_{C, n}\right) \cap \overline{\pi(B \cap(X \times \bar{C}))}=0$. Note that $B \cap\left(\left(\mathrm{cl}_{X} D_{C, n}\right) \times \bar{C}\right)=0$, since if $\langle x, y\rangle \in B \cap(X \times \bar{C})$ then $x \in Z$, for otherwise

$$
\left.\langle x, y\rangle \in\left[\left(\overline{\pi\left(A \cap\left(E_{C} \times \bar{C}\right)\right.}\right) \cap \overline{\pi(B \cap(X \times \bar{C}))}\right) \times \bar{C}\right] \cap G
$$

contradicting the fact that this intersection is empty. So $x \in Z$ and $x \notin \operatorname{cl}_{Z} D_{C, n}$; hence $x \notin \mathrm{cl}_{x} D_{C, n}$.

Define $D_{n}^{m}=\bigcup\left\{D_{C, n} \times C: C \in \mathscr{C}_{m}\right\} \cap G$, and note that $\left(\mathrm{cl}_{G} D_{n}^{m}\right) \cap B=0$ since $\mathscr{C}_{m}$ is locally finite in $\bigcup \mathscr{C}_{m} \supset Y_{0}$. Then, to finish the proof it suffices to show that $\left\{D_{n}^{m}: 0 \leqslant n \leqslant m\right\}$ covers $A$.

So let $\langle x, y\rangle \in A$ and fix $n \in \omega$ and $W_{0} \times W_{1} \subset G$, a neighborhood of $\langle x, y\rangle$ such that $\left(W_{0} \times W_{1}\right) \cap \operatorname{cl}_{G}\left(V \cup U_{n} \cup B\right)=0$. There are $m \geqslant n$ and $C \in \mathscr{C}_{m}$ with $y \in C \subset \bar{C} \subset W_{1}$, so for this $C, x \in Z$, hence $\langle x, y\rangle \in D_{C, n} \times C$. 
3.5. Lemma. If $A, B$ are disjoint closed subsets of $G$ with $A \subset X \times Y_{0}$, then $A, B$ can be separated by open sets.

Proof. By Lemma 3.3, for $i>0$ find an open $U_{i} \supset B \cap\left(X \times Y_{i}\right)$ such that $A \cap \mathrm{cl}_{G} U_{i}=0$. Then, by Lemma 3.4 find an open $U_{0} \supset B \backslash \bigcup_{i>0} U_{i}$ with $A \cap \mathrm{cl}_{G} U_{0}=0$. This shows $A, B$ are separated since $G$ is countably paracompact.

Now we finish the proof. Let $A, B$ be two disjoint closed subsets of $G$. By Lemma 3.3, for $i>0$ fix an open $U_{i} \supset A \cap\left(X \times Y_{i}\right)$ with $B \cap \mathrm{cl}_{i} U_{i}=0$. Then by Lemma 3.5 fix an open $U_{0} \supset A \backslash \bigcup_{i>0} U_{i}$ with $B \cap \mathrm{cl}_{i} U_{0}=0$. This shows that $A$ and $B$ are separated.

3.6. Corollary. Assume $X$ is hereditarily normal and hercilitarily countably paracompact, $Y$ Lašnev, and $G$ an open subset of $X \times Y$. Then $G$ is countably paracompact iff $G$ is normal.

4. Paracompactness. In this section we consider hereditarily collectionwise normal spaces $X$ and show that every normal open subspace $G$ of $X \times Y$, where $Y$ is a Lašnev space, is collectionwise normal, and paracompact if $X$ is hereditarily paracompact. Unlike in the case $G=X \times Y$ we cannot use test spaces ([1], [18]) to get a short proof.

4.1. THEOREM. Assume $X$ is hereditarily collectionwise normal, $Y$ Lašnev, and $G$ an open subset of $X \times Y$. If $G$ is normal then $G$ is collectionwise normal.

Proof. The argument we give is a combination of the proofs of Theorems 3.1 and 3.2. Let $Y=\bigcup_{i \geqslant 0} Y_{i}$, where $Y_{0}=\left\{y \in Y: f^{-1}(y)\right.$ is compact $\}$ and $Y_{i}$ is closed. discrete for $i>0$. Also let $Y_{0}^{\prime}=\left\{y \in Y_{0}: y\right.$ is nonisolated in $\left.Y_{0}\right\}$.

4.2. Lemma. Assume $\left\{H_{\alpha}: \alpha \in \chi\right\}$ is a discrete fumily of closed subsets of $G$ such that $\bigcup\left\{H_{\alpha}: \alpha \in x\right\} \subset X \times Y_{0}^{\prime}$. Then there is a discrete open family $\left\{U_{\alpha}: \alpha \in \varkappa\right\}$ with $H_{\alpha} \subset U_{\alpha}$ for $\alpha \in \varkappa$.

Proof. Since $G$ is normal, it is enough to find a disjoint open family $\left\{U_{\alpha}: \alpha \in \chi\right\}$ with $H_{\alpha} \subset U_{\alpha}$. We work towards an application of Lemma 2.4 .

Let $\left\{\mathscr{C}_{n}: n \in \omega\right\}$ be as in the proof of Lemma 3.4. Let $\mathscr{B}_{n}=\left\{C \cap Y_{0}: C \in \mathscr{C}_{n}\right\}$, $n \in \omega$, and we may assume that if $C \neq C^{\prime} \in \mathscr{C}_{n}^{\prime}$, then $C \cap Y_{0} \neq C^{\prime} \cap Y_{0}$. Hence for $B \in \mathscr{B}_{n}$ there is exactly one $C \in \mathscr{C}_{n}$ with $B=C \cap Y_{0}$ and denote this $C$ by $C_{B}$.

For $n \in \omega$ let $\mathscr{B}_{n}^{\prime}=\left\{B \in \mathscr{B}_{n}: B\right.$ is nondiscrete in $\left.Y_{0}\right\}$ and $\mathscr{B} \mathscr{B}^{\prime}=\bigcup \mathscr{B} \mathscr{B}_{n}^{\prime}$. For $B \in \mathscr{B}^{\prime}$ pick points $p_{B}, q_{B} \in B$ so that no point is picked twice.

Let $\pi: X \times Y \rightarrow X$ be the projection map. For $B \in \bigcup \mathscr{B}{ }_{n}$ let $O_{B}$ be the maximal open set in $X$ such that $O_{B} \times \bar{C}_{B} \subset G$ and $\left\{\pi\left(H_{\alpha} \cap\left(O_{B} \times \bar{C}_{B}\right)\right): \alpha \in \chi\right\}$ is discrete in $O_{B}$. Let $P_{B}=X \backslash O_{B}$, and define sets $H, K$ as in Theorem 3.1. Since $\left.\left\{\mathscr{B}{ }_{n}: n \in \omega\right)\right\}$ is a strong development for $Y_{0}$, one shows exactly as in Theorem 3.1 that $H, K$ are disjoint, closed subsets of $\left(X \times Y_{0}\right) \cap G$. Let $U=G \backslash \cup H_{\alpha}$, and let $T, V, W$ be as in Theorem 3.1.
For $B \in \mathscr{B}_{n}^{\prime}$ let

$$
F_{B}=\left(X \backslash \pi\left(T \cap\left(X \times \bar{C}_{B}\right)\right)\right) \backslash\left(\pi\left(V \cap\left(X \times \bar{C}_{B}\right)\right) \cap \pi\left(W \cap\left(X \times \bar{C}_{B}\right)\right)\right) .
$$

We show that

$$
O_{B} \times\left(B \cap Y_{0}^{\prime}\right) \supset\left(F_{B} \times\left(B \cap Y_{0}^{\prime}\right)\right) \cap(G \backslash T) .
$$

To see this let $\langle x, y\rangle \in\left(F_{B} \times\left(B \cap Y_{0}^{\prime}\right)\right) \cap G$; then $\left(\{x\} \times \bar{C}_{B}\right) \cap T=0$. Assume $x \notin O_{B}$ and let $m \geqslant n$ be the least such that there is a $D \in \mathscr{B}_{m}^{\prime}$ with $D \subset B$ and a neighborhood $S$ of $x$ with $S \times \bar{C}_{D} \subset G$ so that $S \times \bar{C}_{D}$ intersect at most one of the $H_{\alpha}$ 's. Such $D$ exists; then $K \cap(\{x\} \times D) \neq 0$ and $H \cap(\{x\} \times D) \neq 0$. But then $(K \backslash T) \cap(\{x\} \times D) \neq 0$ and $(H \backslash T) \cap(\{x\} \times D) \neq 0$, so

$$
x \in \pi\left(V \cap\left(X \times \bar{C}_{B}\right)\right) \cap \pi\left(W \cap\left(X \times \bar{C}_{B}\right)\right)
$$

contradicting the fact $x \in F_{B}$.

The set $O_{B} \cap F_{B}$ is collectionwise normal so fix a family $\left\{D_{B, x}: \alpha \in \chi\right\}$ open and discrete in $O_{B} \cap F_{B}$ so that $\pi\left(H_{\alpha} \cap\left(\left(O_{B} \cap F_{B}\right) \times \bar{C}_{B}\right)\right) \subset D_{B, \alpha}$ and

$$
\left(\mathrm{cl}_{O_{B \cap F_{B}}}\left(\bigcup_{\beta \neq \alpha} D_{B, \beta}\right)\right) \cap \pi\left(H_{\alpha} \cap\left(\left(O_{B} \cap F_{B}\right) \times \bar{C}_{B}\right)=0\right.
$$

for $\alpha \in \varkappa$.

We show that for every $\alpha \in \chi$,

$$
\left(\left(\mathrm{cl}_{X}\left(\bigcup_{\beta \neq \alpha} D_{B, \beta}\right)\right) \times C_{B}\right) \cap H_{\alpha}=0 .
$$

Assume not and let $\langle x, y\rangle$ be in the above intersection. So $y \in C_{B} \cap Y_{0}^{\prime}=B \cap Y_{0}^{\prime}$ and $x \in F_{B}$ because $\operatorname{cl}_{X}\left(\bigcup_{\beta \neq \alpha} D_{B, \beta}\right) \subset F_{B}$. Then by $(*) x \in O_{B}$; hence

$$
x \notin \operatorname{cl}_{O_{B \cap F_{B}}}\left(\bigcup_{\beta \neq \alpha} D_{B, \beta}\right)=O_{B} \cap F_{B} \cap \mathrm{cl}_{X}\left(\bigcup_{\beta \neq \alpha} D_{B, \beta}\right) .
$$

For $n \in \omega$ let $\left\{E_{B}: B \in \mathscr{B}_{n}\right\}$ be a shrinking of $\left\{C_{B}: B \in \mathscr{B}_{n}\right\}$ covering $\cup \mathscr{C}_{n}$. For $n \in \omega$ and $\alpha \in \varkappa$ define

$$
D_{\alpha}^{n}=\bigcup\left\{\left(\operatorname{int}_{X} D_{B, \alpha}\right) \times E_{B}: B \in \mathscr{B}_{n}^{\prime}\right\} \cap G .
$$

Observe that since $\mathscr{C}_{n}$ is locally finite in $\bigcup \mathscr{C}_{n} \supset Y_{0}$ we have $H_{\alpha} \cap \mathrm{cl}_{G}\left(\bigcup_{\beta \neq \alpha} D_{\beta}^{n}\right)=0$ so to finish the proof it suffices to show $H_{\alpha} \subset \bigcup_{n \in \omega} D_{\alpha}^{n}$ for $\alpha \in x$.

Let $\langle x, y\rangle \in H_{\alpha}$ and assume $\langle x, y\rangle \notin \mathrm{cl}_{G} V$. Let $W_{0} \times W_{1}$ be an open neighborhood of $\langle x, y\rangle$ with $W_{0} \times W_{1} \subset G$ and $\left(W_{0} \times W_{1}\right) \cap\left(T \cup V \cup\left(\cup H_{\beta}\right)\right)=0$. There is an $n \in \omega$ with $\operatorname{St}\left(y, \mathscr{C}_{n}\right) \subset W_{1}$, so for every $C \in \mathscr{C}_{n}$ containing $y$ we have that $x \in \operatorname{int}_{X}\left(O_{B} \cap F_{B}\right)$ where $B=C \cap Y_{0} \in \mathscr{B}_{n}^{\prime}$. Pick an $E_{B}$ with $y \in E_{B}$; then $\langle x, y\rangle \in\left(\operatorname{int}_{X} D_{B, \alpha}\right) \times E_{B}$ so $\langle x, y\rangle \in D_{\alpha}^{n}$. 
Let $\left\{H_{\alpha}: \alpha \in x\right\}$ be a discrete closed family in $G$. Let $i>0$; since $Y_{l}$ is closed and discrete and $G$ normal there is a discrete open family $\left\{U_{\alpha}^{i}: \alpha \in Y_{i}\right.$; with $H_{\alpha} \cap\left(X \times Y_{i}\right) \subset U_{\alpha}^{i}$ and $\left(\operatorname{cl}_{G} U_{\alpha}^{i}\right) \cap\left(\bigcup H_{\beta}\right)=0$. Let $U=\bigcup\left\{U_{\alpha}^{i}: \alpha \in \varkappa \wedge i>0\right\}$.

Let $D$ be a closed discrete subset of $Y_{0}$. Since $X, Y$ are hereditarily collectionwise normal there is a disjoint open family $\left\{V_{\alpha}^{\prime}: \alpha \in X_{\}}^{\prime}\right.$ in $G$ such that $H_{\alpha} \cap(X \times D) \subset V_{\alpha}^{\prime}$ for $\alpha \in \chi$. Since $G$ is normal and $D$ closed in $Y_{0}$ one can pick open set $V_{\alpha}$ in $G$ so that $\left(H_{\alpha} \cap(X \times D)\right) \backslash U \subset V_{\alpha}, \operatorname{cl}_{G} V_{\alpha} \subset V_{\alpha}^{\prime} \backslash\left(\bigcup_{\beta \neq \alpha} H_{\beta}\right)$, and $\left\{V_{\alpha}: \alpha \in x\right\}$ is discrete in $G$. Since $Y_{0} \backslash Y_{0}^{\prime}$ is a countable union of closed discrete subsets of $Y_{0}$ we can find open sets $V_{\alpha}^{t}, \alpha \in \varkappa, i \in(\omega)$, so that

$$
\left(H_{\alpha} \cap\left(X \times\left(Y_{0} \backslash Y_{0}^{\prime}\right)\right)\right) \backslash U \subset \bigcup_{i \in(1)} V_{\alpha}^{i}
$$

$\operatorname{cl}_{G} V_{\alpha}^{i} \cap\left(\bigcup_{\beta \neq \alpha} H_{\beta}\right)=0$, for $\alpha \in \mathcal{x}$, and $\left\{V_{\alpha}^{i}: \alpha \in x_{\}}\right\}$is discrete for $i \in \omega$. Let $V=\bigcup\left\{V_{\alpha}^{i}: i \in \omega \wedge \alpha \in \chi\right\}$.

By Lemma 4.2 there is a discrete open family $\left\{W_{\alpha}: \alpha \in \mathcal{X}\right\}$ with $H_{\alpha} \backslash(U \cup V) \subset W_{\alpha}$ and $\mathrm{cl}_{G} W_{\alpha} \cap\left(\bigcup_{\beta \neq \alpha} H_{\beta}\right)=0$ for $\alpha \in \chi$.

Then by Lemma 2.4, $\left\{H_{\alpha}: \alpha \in x\right\}$ can be separated.

4.3. Theorem. Assume $X$ is hereditarily paracompact, $Y$ Lašnev, and $G$ a normal open subspace of $X \times Y$. Then $G$ is paracompact.

Proof. Observe that a collectionwise normal space $Z$ is paracompact if every open cover has a $\sigma$-locally finite closed refinement. To see this, first note that $Z$ is countably paracompact and then use $[10,16$, also $11,5.5 .18 \mathrm{a})]$ to extend this refinement to a $\sigma$-locally finite open refinement.

Since $G$ is collectionwise normal by Theorem 4.2 we find a $\sigma$-locally finite closed shrinking of the cover $\left\{U_{\alpha}: \alpha \in \chi\right\}$. This is done almost exactly as in Theorem 3.1, so we use the same notation. Only difference is that instead of arbitrary shrinkings we take locally finite shrinkings, and again by $[10,16$, i.e. $11,5.5 .18 \mathrm{a})]$ we can have $\left\{U_{\alpha}^{i}: \alpha \in x\right\}, i>0$, locally finite and also extend $\left\{A_{\alpha}^{i} \backslash U: \alpha \in \mathcal{X}\right\}$ to a locally finite open family for $i \in \omega$. Finally, take $\left\{Q_{B, \alpha}: \alpha \in X\right\}$ to be a locally finite closed family in $O_{B} \cap F_{B}$.

5. $M P$-spaces. In this section we consider products of $P$-spaces with $M$-spaces and show that in some cases normality of the product is equivalent to its countable paracompactness.

5.1. THeorem. Assume $X$ is a first countable, paracompact $P$-space, $Y$ is the closed image of a normal $M$-space, and $X \times Y$ is countably paracompact. Then $X \times Y$ is normal.

Proof. Let $Z$ be a normal $M$-space, $f: Z \rightarrow Y$ a closed map, and $\left\{Y_{i}: i \in \omega\right\}$ as in Lemma 2.1. We use the same idea as in the proof of Theorem 3.2.

5.2. LEMMA. If $A, B$ are disjoint closed subsets of $X \times Y$ such that $A \subset X \times Y_{i}$ for some $i>0$ then $A, B$ are separated by open sets.
Proof. Note that $Y$ is collectionwise normal since every normal $M$-space is collectionwise normal.

For $y \in Y_{i}$ fix an open $H_{y}$ containing $y$ such that $\left\{H_{y}: y \in Y_{i}\right\}$ is discrete, and let $\left\{V \times U_{y}^{V}: V \in \mathscr{V}_{y}\right\}$ be an open cover of $X \times\{y\}$ such that each $U_{y}^{V} \subset H_{y}, \mathscr{V}$, is locally finite in $X$, and the closure of each $V \times U_{y}^{V}$ intersects at most one of $A$ and $B$. Then

$$
W=\bigcup\left\{V \times U_{y}^{V}: y \in Y_{i} \wedge V \in \mathscr{V}_{y} \wedge\left(V \times U_{y}^{V}\right) \cap A \neq 0\right\}
$$

covers $A$ and $\bar{W} \cap B=0$

5.3. Lemma. If $A, B$ are closed disjoint subsets of $X \times Y$ such that $A \subset X \times \underset{i>0}{\cup} Y_{i}$ then they can be separated by open sets.

Proof. Using Lemma 5.2 fix open sets $U_{i} \supset A \cap\left(X \times Y_{i}\right)$ for $i>0$ such that $\bar{U}_{i} \cap B=0$. Since $X \times Y$ is countably paracompact, $A$ and $B$ can be separated by open sets.

5.4. Lemma. If $A, B$ are closed disjoint subsets of $X \times Y$ with $A \cup B \subset X \times Y_{0}$ then $A, B$ can be separated by open sets.

Proof. Since every normal $M$-space is a collectionwise normal $\Sigma$-space (Lemmas 2.5 and 2:6), $X \times Z$ is normal by Lemma 2.9. So there are disjoint open subsets $U, V$ of $X \times Z$ such that $\left(\mathrm{id}_{X} \times f\right)^{-1}(A) \subset U$ and $\left(\mathrm{id}_{X} \times f\right)^{-1}(B) \subset V$. Using the fact that $f^{-1}(y)$ is countably compact for $y \in Y_{0}$ and $X$ is first countable, it is easy to check that $A \subset \operatorname{int}\left[\left(\operatorname{id}_{X} \times f\right)^{\#}(U)\right]$ and $B \subset \operatorname{int}\left[\left(\operatorname{id}_{X} \times f\right)^{\#}(V)\right]$, and of course

$$
\operatorname{int}\left[\left(\operatorname{id}_{X} \times f\right)^{\#}(V)\right] \cap \operatorname{int}\left[\left(\operatorname{id}_{X} \times f\right)^{\#}(U)\right]=0 .
$$

The same argument as in Theorem 3.2 finishes the proof

5.5. THEOREM. Assume $X$ is a first countable, subparacompact P-space, $Y$ a $x$-shrinking $\Sigma$-space, and $X \times Y$ normal. Then $X \times Y$ is $\chi$-shrinking.

Proof. By Lemma 2.2 and the fact that $X \times Y$ is normal it suffices to show the following

5.6. Lemma. Assume $X$ is a first countable, subparacompact $P$-space, and $Y$ a $x$-shrinking $\Sigma$-space. Then every open cover of $X \times Y$ of size $x$ has a closed $\sigma$-shrinking.

Proof. Let $\left\{\mathscr{F}_{m}: m \in \omega\right\}$ be a family in $Y$ satisfying the conditions of Lemma 2.7. Let $\left\{G_{\alpha}: \alpha \in \varkappa\right\}$ be an open cover of $X \times Y$.

For $s \in \lambda^{<\omega}$ let $\left\{U_{\beta} \times V_{\beta, \alpha}: \beta \in I(s) \wedge \alpha \in \chi\right\}$ be a maximal open family in $X \times Y$ such that every $U_{\beta} \times V_{\beta, \alpha} \subset G_{\alpha}$ and $F(s) \subset \bigcup_{\alpha \in X} V_{\beta, \alpha}$ for $\beta \in I(s)$. (Here $I(s)$ is an index set depending on $s$.)

Define $H(s)=\bigcup\left\{U_{\beta}: \beta \in I(s)\right\}$ and note that $H(s) \subset H(\hat{s}\langle\alpha\rangle)$ for $\alpha \in \lambda$. So, since $X$ is a $P$-space, there are closed $K(s) \subset H(s)$ so that $\bigcup_{n \in(t)} K(f \uparrow n)=X$ whenever $\bigcup_{n \in \omega} H\left(f\lceil n)=X\right.$ for $f \in \lambda^{\omega}$.

CLAIM. $\left\{K(s) \times F(s): s \in \lambda^{<(0)}\right\}$ cover $X \times Y$. 
Proof. We first show that for every $\langle x, y\rangle \in X \times Y$ and $\alpha \in \varkappa$ there is a neighborhood $U \times V_{\alpha}$ of $\langle x, y\rangle$ with

$$
U \times V_{\alpha} \subset G_{\alpha} \text { and } C(\nu) \subset \bigcup_{\alpha \in x} V_{\alpha} .
$$

To see this fix a decreasing basis $\left\{U_{n}: n \in \omega\right\}$ for $x$. Let $V_{n, \alpha}$ be the maximal open set in $Y$ with $U_{n} \times V_{n, \alpha} \subset G_{\alpha}$ and define $V_{n}=U\left\{V_{n, \alpha}: \alpha \in \mathcal{X}\right\}$. Then $\left\{V_{n} ; n \in \omega\right\}$ is an increasing open eover of the countably compact $C(y)$ (Lemma $2.7\left(\right.$ iv)) so fix an $n \in \omega$ with $V_{n} \supset C(y)$. For this $n$ let $U=U_{n}$ and $V_{\alpha}=V_{n, \alpha}$.

For $y \in Y$ let $f \in \lambda^{\omega}$ be such that $\{F(f \mid n): n \in(1)\}$ is a net for $(y)$. By $(*)$ we have that $\bigcup_{n \in(\omega)} H(f \vdash n)=X$, so if $x \in X$ there is an $n$ with $x \in K(f \uparrow n)$; hence $\langle x, y\rangle \in K(f \uparrow n) \times F(f \uparrow n)$

Since $K(s)$ is subparacompact and $K(s) \subset U\left\{U_{\beta}: \beta \in I(s)\right\}$ let

$$
\left\{M_{\beta, n}^{\prime \prime}: \beta \in I(s) \wedge n \in(\omega)\right\}
$$

be a $\sigma$-discrete closed $\sigma$-shrinking of $\left\{U_{\beta}: \beta \in I(s)\right\}$. Also, for $\beta \in I(s)$ let $\left\{N_{\beta, \alpha}: \alpha \in x_{\}}\right\}$ be a closed shrinking of $\left\{V_{\beta, \alpha}: \alpha \in x\right\}$ covering $F(s)$.

For $m, n \in \omega$ and $\alpha \in \chi$ let

$$
T_{m, n}^{\alpha}=\bigcup\left\{M_{\beta, n} \times N_{\beta, \alpha}: \beta \in I(s) \wedge s \in S_{m}\right\} .
$$

Since $\mathscr{F}_{m}$ is locally finite and $\left\{M_{\beta, n}: \beta \in I(s)\right\}$ discrete, each $T_{m, n}^{\alpha \alpha}$ is closed. This gives us a closed $\sigma$-shrinking of $\left\{G_{\alpha}: \alpha \in x\right\}$.

5.7. Corollary. Assume $X$ is a first countable, subparacompact P-space, $Y$ a $\Sigma$-space, and $X \times Y$ normal. Then $X \times Y$ is countably paracompact.

5.8. Theorem. Assume $X$ is a first countable, subparacompact $P$-space, $Y$ the closed image of a $x$-shrinking $M$-space, and $X \times Y$ normal. Then $X \times Y$ is $x$-shrinking.

Proof. Let $Z$ be a $x$-shrinking $M$-space, $f: Z \rightarrow Y$ a closed map, and $\left\{Y_{i}: i \in \omega\right\}$ as in Lemma 2.1 Let $\left\{G_{\alpha}: \alpha \in X\right\}$ be an open cover of $X \times Y$. We find a closed $\sigma$-shrinking of $\left\{G_{\alpha}: \alpha \in \chi\right\}$ and thus show $X \times Y$ is $x$-shrinking (Lemma 2.2).

For $i>0$, since $X \times Y_{i}$ is $x$-shrinking, there are closed subsets $K_{\alpha, l}$ of $X \times Y_{i}$ covering $X \times Y_{i}$ such that $K_{\alpha, i} \subset G_{\alpha}$ for $\alpha \in \chi$. Since $X \times Y$ is normal fix open sets $H_{\alpha, i} \supset K_{\alpha, i}$ with $\bar{H}_{\alpha, i} \subset G_{\alpha}$. Let $H=\bigcup\left\{H_{\alpha, i}: \alpha \in \varkappa \wedge i>0\right\}$ and $E=(X \times Y) \backslash H$. So $E$ is closed and $E \subset X \times Y_{0}$.

To finish the proof it suffices to cover $E$. Let $\psi=\left(\mathrm{id}_{X} \times f\right): X \times Z \rightarrow X \times Y$. By Lemma 5.6 there is a closed $\sigma$-shrinking $\left\{F_{\alpha, n}: \alpha \in x \wedge n \in \omega\right\}$ of $\left\{\psi^{-1}\left(C_{\alpha}^{i}\right): \alpha \in x_{\}}\right.$. Since $\psi \uparrow X \times f^{-1}\left(Y_{0}\right)$ is closed by Lemma 2.8 , we have that

$$
\left\{\psi\left(F_{\alpha, n}\right) \cap E: \alpha \in \psi \wedge n \in(\omega)\right\}
$$

is a closed cover of $E$ satisfying $\psi\left(F_{\alpha, n}\right) \cap E \subset G_{\alpha}$.

5.9. Corollary. Assume $X$ is a first countable paracompact $P$-space and $Y$ is the closed image of a $x$-shrinking $M$-space. Then the following are equivalent:

(i) $X \times Y$ is normal, (ii) $X \times Y$ is c'ountably paracompact,

(iii) $X \times Y$ is $x$-shrinking,

(iv) $X \times Y$ is collectionwise normal.

Proof. Theorems 5.1 and 5.8 show that (i), (ii), and (iii) are equivalent (recall that $x$ is infinite). The equivalence of (i) and (iv) is shown in [8, Theorem 1].

5.10. THEOREM, Let $X$ be a space which can be represented as the union of two disjoint sets $C$ and $D$ such that the points in $C$ are isolated in $X$ and $D$ is discrete. Assume $Y$ is shrinking and $X \times Y$ normal. Then $X \times Y$ is shrinking.

Proof. Like in the proof of Theorem 5.8 first cover $D \times Y$, and get an open set $H \supset D \times Y$. Since the points in $C$ are isolated it is easy to cover $(X \times Y) \backslash H$.

Above contains Theorem 5 from [34]. Note also that any subspace of Bing's $G$ and $H$ satisfies the conditions of Theorem 5.10.

5.11. EXAMPLES. We now show that the product of a first countable paracompact $P$-space with a $\Sigma$-space need not be countably paracompact, and also that it can be countably paracompact but not normal. So the assumptions in Corollary 5.7 are necessary and the converse of the corollary does not hold.

Let $X$ be any first countable compact space of weight $2^{\omega}$ and let $Y$ be Bing's $H$ [4]. Then $Y$ is a countably paracompact $\Sigma$-space, so $X \times Y$ is countably pracompact. To see that $X \times Y$ is not normal apply either [27, Theorem 2] (for a short proof see $[32,3.1]$, also $[25,3.12]$ or $[6$, page 6$]$, also $[9$, Corollary 6]).

Now we construct a non-normal, non-countably paracompact product $X \times Y$. Let $X$ be the space from Example 9.1 in [5]. This $X$ is first countable and stratifiable so $X$ is perfectly normal (and hence $P$ ) and paracompact (see [12]). Let $Y$ be Bing's $H$ [4], so $Y$ is a normal $\Sigma$-space. It is shown in [6, Example 1] that $X \times Y$ is not normal. Since $X$ is a paracompact $\sigma$-space and $Y$ a normal $P$-space, [13, Theorem 3.1] shows that $X \times Y$ is not countably paracompact, for otherwise it would be normal.

\section{References}

[1] O. T. Alas, On a characterization of collectionwise normality, Canad. Math. Bull. 14 (1971), 13-15.

[2] A. Bešlagić, Normality in products, Topology Appl., 22 (1986), 71-82.

[3] - A Dowker product, Trans. Amer. Math. Soc., 292, No 2 (1985), 519 530.

[4] R. H. Bing, Metrization of topological spaces, Canad. J. Math. 3 (1951), 175-186.

[4] R. H. Bing, Metrization of topological spaces, Canad. J. Macic J. Math. 11 (1961), 105-125.

[6] K. Chiba, On products of normal spaces, Rep. Fac. Sci. Shizuoka Univ. 9 (1974), 1-11.

[7] -, On collectionwise normality of product spaces, I, Proc. Amer. Math. Soc. 91 (1984), 649-652.

[8] -, On collectionwise normality of product spaces, Ir, Proc. Amer. Math. Soc. 91 (1984), $653-657$.

[9] K. Chi ba, T. C. Przymusi ński and M. E. Rudin, Normality in product spaces and Morita's conjectures, Topology Appl. 22 (1986), 19-32.

[10] C. H. Dowker, Homotopy extension theorems, Proc. London Math. Soc. 6 (1956), 100-116.

[11] R. Engelking, General Topology, Polish Scientific Publishers, Warszawa 1977. 


\section{More on distributive ideals}

by

\section{C.A. Johnson (Kecle)}

Abstract. In this paper we present further results concerning ideals on uncountable cardinals whose quotient algebra is distributive. We show that such ideals are related to completely ineffable and weakly compact cardinals, flipping properties, $V$-ultrafilters, ideal theoretic partition relations and a closure property of the generic ultrapower.

In [14] we commenced our study of ideals on uncountable cardinals whose quotient algebra is distributive, and in particular we showed that distributivity is related to some ideal theoretic partition propertics. In this paper we present some further results concerning such "distributive ideals", and whilst for the most part not strictly necessary, a familiarity with [14] would be useful.

In $\S 1$ we show that if $x$ is completely ineffable then $x$ carries a natural normal $(\varkappa, x)$-distributive ideal, the completely ineffable ideal. It is a well-known question whether natural normal ideals (especially the non-stationary ideal) can ever be saturated (or precipitous). We answer this question for the completely ineffable ideal by showing it to be non-precipitous.

$\$ 2$ contains some brief remarks connecting distributive ideals to $V$-ultrafilters and flipping properties. Using distributivity, we also give a simple proof of a theorem of Kleinberg [18] characterizing completely ineffable cardinals in terms of the existence of certain $V$-ultrafilters.

In $\S 3$ we make some further remarks concerning normal WC ideals. It follows easily from results of Baumgartner [2] and of [14] that the existence of a normal WC ideal on $x$ is equivalent to the weak compactness of $x$. Indeed, the existence of such an ideal may be regarded as a (normal) ideal theoretic analogue of the "strong inaccessibility and tree property" equivalent of weak compactness. This ideal theoretic analogue is shown to have considerable power easily yielding $\Pi_{1}^{1}$-indescribability and a combinatorial equivalent of weak compactness due to Shelah [21].

In $\S 4$ various forms of weak distributivity are considered, and are shown to be related to ideal theoretic versions of partition relations akin to those defining Rowbottom cardinals. Also, using a forcing argument similar to that of [14, Theorem 9], we give a new proof of a partition theorem for saturated ideals originally due to Solovay.

In $\$ 5$ we briefly mention a connection between distributivity and a closure property of the generic ultrapower.

§ 0. Notation and terminology. Our set-theoretical terminology is reasonably standard (see [11]), and background results, notation and terminology not defined here concerning ideals may be found in [5], [12] or [14].

Lower case greek letters will denote ordinals and when a set of ordinals is written as $\left\{\zeta_{1}, \zeta_{2}, \ldots, \zeta_{n}\right\}$ it is assumed that $\zeta_{1}<\zeta_{2}<\ldots<\zeta_{n}$ and if $m \leqslant n$ then $\left\{\zeta_{1}, \zeta_{2}, \ldots, \zeta_{n}\right\} \mid m=\left\{\zeta_{1}, \zeta_{2}, \ldots, \zeta_{m}\right\}$. Throughout the paper $x$ will denote a regular uncountable cardinal, and $I$ a proper non-principal $x$-complete ideal on $x$ (see 